\title{
ARRAY COVARIANCE ERROR MEASUREMENT IN ADAPTIVE SOURCE PARAMETER ESTIMATION.
}

\author{
Ana Pérez-Neira, M.A. Lagunas
}

TSC Dept., E.T.S.I. Tolecom.

Apdo.30002. 08080 Barcelona. SPAIN

\begin{abstract}
The small error approximation is used to derive a linear relationship between the source parameters (i.e. power levels and directions of arrival or DOAs) and a measurement of the covariance error matrix, defined as the difference between a nonparametric consistent estimate of the spectral density matrix and a covariance model from the scenario parameters (source strength and DOA). The resulting framework allows the design of a Kalman like algorithm which provides a simultaneous and adaptive estimation of the source parameter no matter what is the source waveform or modulation. Due to its similarities with the Kalman filter structure [1] good performance is expected, mainly in presence of sensors misfunctioning, low signal-to-noise ratio (SNR),etc...
\end{abstract}

\section{INTRODUCTION}

The problem of obtaining information about the features of the waves impinging an aperture of sensors has received considerable attention. It is well-known that the Extended Kalman Filter (digital version of classical PLLs) is used to overcome the problems related to the estimation of the temporal features of a signal in noise. It is widely used for time reference regeneration because of its istability in simultaneously estimate the frequency, phase and magnitude of a signal even for non-stationary situations. If, on the contrary, we are interested in the spatial features of a signal, a simultaneous and adaptive estimation of DOA and source level still remains an important problem to solve and this will be the main concern of this work.

The study of DOA estimation in array processing has been faced from the spectral estimation point of view. Due to the random nature of the snapshot, the spectral covariance matrix has been always the starting point and the modeling objective. It will contain the required information of the power and location of the sources in the scene. The Maximum Likelihood Estimation technique (MLE) was one of the first ones to be investigated. It provides optimum results but it involves a complicated non-linear optimization problem. that is the reason why suboptimal techniques with a reduced computational load have dominated the field. These procedures usually consist of two steps: some procedures perform an nitial estimate using high-resolution methods. then enhance this estimate by means of an optimum adaptive beamformer or by means of an iterative process [2,3]. Another possibility is to obtain the source location by gradient search techniques, then the maximum likelihood of the source strength proceeds $[4,5,6]$. The main drawback of all these techniques is their computational cost and their incapability of tracking many sources.

We have mentioned before that digital PLLs show a high perfomance in the time scenario, for this reason a way out of our problem may be obtained if it we find the spatial counterpart of these temporal parameter estimation techniques. This implies the application of a state space filter that acts on the spectral covariance matrix in order to solve a spatial reference regeneration problem. This time-space analogy is possible because the point source model we use for signals received in an aperture scenario promotes, in a narrow band approach, a pure frequency in the spatial domain, known as the steering vector, which is associated to the corresponding DOA.

The new approach discussed in this paper is to adress the problem of DOA and power estimation from a spectral point of view (whose starting point is the spectral covariance matrix) through the use of a linear adaptive filter to solve it. The objective of this EKF-style algorithm will be the minimization of some kind of measurement error: this will be taken from the covariance error matrix. On the other hand, this error should be linear dependent on the source parameter to be estimated, thus the small error approximation will be used.

The presentation starts by showing to what extent the criteria of minimizing the covariance error is related to the MLE. Then, the measurement error is reported, proving that it supports the implementation of the resulting linear adaptive filter. This one will be finally presented in section 4 together with some of the results obtained.

\section{THE ML ESTIMATE AND THE ARRAY COVARIANCE ERROR}

In the frequency domain, the array snapshot can be modeled as follows: 


$$
X(w)=A(w) \cdot S(w)+\underline{U}(w)
$$

where $A(w)$ is the matrix formed by the steering vectors asn, which contains the DOA of each source $s$ at time $n$, $\mathbf{S}(w)$ is the Fourier-Transformed signals impinging the aperture's origin and $\underline{U}(W)$ is the white gaussian sensors' noise, which we assume incorrelated with the signals. The spectral matrix of the array output can be expressed as:

$$
\underline{\underline{C}} x(w)=\stackrel{A}{=}(w) \cdot \underline{\underline{C}} s(w) \cdot \stackrel{A}{=}^{H}(w)+\sigma^{2} \cdot !
$$

where $\underline{G}(w)$ is the spectral matrix of the signals and $\sigma^{2}$ is the noise power.

If we apply to the asymptotic properties of the FourierTransformed array output in (1), the maximum likelihood estimates can be formulated as indicated in (3):

$$
L(E)=-\log \operatorname{det} \underline{\underline{C}} \times(E)-\operatorname{tr}\left(\underline{\underline{C}} \cdot \underline{\underline{C}} \times(\underline{E})^{-1}\right)
$$

where $\underline{\underline{C}} \times(E)$ is the estimated covariance matrix and $\hat{\underline{C_{x}}}$ is the consistent estimate of the actual covariance matrix:

$$
\underline{\underline{E}} x=(1 / K) \cdot \Sigma_{k=1}^{N} \underline{x k} \cdot \underline{x}{ }^{H}
$$

the vector $E$ contains the parameter to estimate: the spectral matrix of the signal,Cs, the DOA's, $\xi$, and the noise power, $\sigma^{2}$ : $\quad E^{\mathrm{T}}=\left(\underline{C} s, \xi, \sigma^{2}\right)$.

In (3), the notation of "w" has been omitted because we are dealing with the narrowband case.

In order to obtain the MLE the function $L\left(\underline{\underline{C}} s, \xi, \sigma^{2}\right)$ will be first maximize over $\underline{\underline{C}}$ and $\sigma^{2}$, keeping the parameter vector $\xi$ fixed. If explicite solutions are found, we put them into the likelihood function (3) and the resulting criterion is maximize over $\xi$. The resulting ML estimates are the one presented in [3]; for the spectral covariance matrix, $\underline{\underline{X}}$ it can be formulated as follows:

$$
\left.\hat{C_{S}}(x)=\underline{\underline{P}} \cdot \underline{\underline{\hat{C}}} x \cdot \underline{\underline{P}} \cdot \wedge^{\wedge} \sigma^{2} \cdot \underline{\underline{A}} \underline{\underline{H}}^{\mathrm{A}}\right)^{-1}
$$

where $\underline{P}$ is the projection matrix onto de DOA subspace:

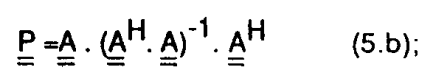

For the noise power the final estimate is the one presented in (6):

$$
\wedge^{2}=(1 / N Q) \cdot \operatorname{tr}\left\{(\underline{1-P}-\underline{\underline{P}}) \cdot \hat{\underline{\hat{C}_{x}}}\right\}
$$

being NQ the number of active sensors of the aperture.
As reported in $[7,8]$ the MLE is the same as the one obtained by the minimization of the quadratic covariance error:

$$
Q(E)=\operatorname{tr}\left\{(\underline{\underline{C}} x(E)-\hat{\underline{C}} x)^{2}\right\}
$$

This criterion is widely used in the literature in order to solve many of the problems that arise in sensor array signal processing. The author d'Assumpcao [9] is one example; by means of the least squares fits criterion in (7) d'Assumpcao designs different estimators for the signals' strengths. Clearly, the key point is the measurement of the covariance error matrix. The way this measurement is made depends on the problem to deal with [10]. If instead of an error matrix an scalar is needed, the correct choice is to consider the norm of the matrix, generally the trace. In our approach, the basic derivations to design our linear estimator in section 4 come from a Kalman-like formulation; and the state space model we use needs that the error measurement fullifils two requirements. On the one hand, it must not be a scalar but an error vector; on the other hand, it must depend linearly on the source paramenters, $E$. The next section reports the way to obtain a covariance error measurement which fulfills the two features mentioned above.

\section{LINEAR STATE SPACE MODEL FOR THE COVARIANCE ERROR MEASUREMENT: THE SMALL ERROR APPROXIMATION.}

Let us assume $\mathrm{N}$ incoherent sources in the scene, the estimation of the parameter vector, at iteration $n$, will be:

$\hat{E}_{n}=\left(c_{1 n}, \hat{c}_{2 n}, \ldots \hat{c}_{N}, \hat{\xi}_{1 n}, \hat{\xi}_{2 n}, \ldots, \xi_{N n}, \wedge \sigma^{2}\right)^{\top}$

The error matrix, in terms of these magnituds is:

$$
\begin{aligned}
& \tilde{\underline{\underline{\underline{C}}}} \mathrm{x}=\underline{\underline{\underline{C}}} x \cdot \underline{\underline{C}} x\left(\hat{E_{n}}\right)= \\
& =\Sigma_{s=1}^{N}\left(c_{s} \cdot \operatorname{asn} \cdot \underline{a} \hat{s} n \cdot c_{s} n \cdot a\left(\xi_{s} \hat{n}\right) \cdot a^{H}(\xi \hat{s} n)\right)+ \\
& +\sigma^{2} !
\end{aligned}
$$

As concerns to the bold-letter part of expression above, this can be expressed as a summatory of the following terms:

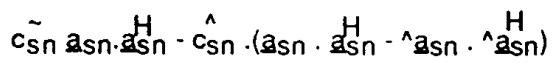

where $\tilde{c_{s}}$ is the source level error:

$$
\tilde{c_{s n}}=c_{s n} \cdot \hat{c_{s n}}
$$

At his moment, the small error approximation will be used in order to linearize the measured covariance error in terms of the error in the parameters. Thus, assuming 
that, at a given iteration $\hat{a s}_{n}$ is close to the actual steering vector, the term in (10) can be approximated by:

$$
\hat{c_{s n}} \hat{a_{s n}} \cdot \hat{a_{s n}^{H}}+\hat{c_{s n}} \cdot\left(\hat{a s} \cdot \hat{a_{s n}^{H}}\right)
$$

naming assn the steering vector error:

$$
\tilde{a}_{s n}=a_{s n}-a\left(\hat{\xi}_{s n}\right)=a_{s n} \cdot \hat{a}_{s n}
$$

In order to achieve the linear relationship between the error measure and the elevation error, the approximation is used again in each components " $q$ " of the steering vector error, aśn

$$
\begin{aligned}
& \left(\tilde{a}_{s}\right)_{q}=\left(a_{s}\right)_{q}-\left(\hat{a_{s}}\right)_{q}=\exp \left(k_{q} \cdot \sin (\xi s)\right)- \\
& -\exp \left(k_{q} \cdot \operatorname{Sin}\left(\hat{\xi}_{s}\right)\right)= \\
& =\exp \left(k_{q} \cdot \operatorname{Sin}\left(\tilde{\xi_{s}}+\hat{\xi}_{s}\right)\right)-\exp \left(k_{q} \cdot \operatorname{Sin}\left(\hat{\xi_{s}}\right)\right) \sim \\
& -\exp \left(k_{q} \cdot\left(\operatorname{Sin} \hat{\xi_{s}}+\tilde{\xi}_{s} \cdot \operatorname{Cos} \hat{\xi}_{s}\right)\right)-\exp \left(k_{q} \cdot \operatorname{Sin}\left(\hat{\xi}_{s}\right)\right) \sim \\
& \sim \exp \left(k_{q} \cdot \operatorname{Sin}\left(\hat{\xi_{s}}\right)\right) \cdot\left(j \cdot k_{q} \cdot \operatorname{Cos}\left(\hat{\xi}_{s}\right) \cdot \tilde{\xi}_{s}\right)
\end{aligned}
$$

$$
\left(\tilde{a}_{s}\right)_{q} \sim\left(\hat{\hat{a}_{s}}\right)_{q} \cdot\left(j \cdot k_{q} \cdot \cos \hat{\xi}_{s} \cdot \tilde{\xi}_{s}\right)
$$

where $k_{q}=(2 \cdot \pi \cdot f / c) \cdot d q \cdot \operatorname{Cos}\left(\phi_{q}\right)$, being "f " the central frequency and $d_{q}$ and $\phi_{q}$ are the distance to the phase center and the azimuth of sensor $q$ (we assume a filled linear array).

In summary, the covariance matrix can be set in a linear relationship with the parmeters errors,

$\tilde{c}_{x}=\Sigma_{s=1}^{N}\left(\tilde{c_{n s}} \cdot \underline{a_{n s}^{n}} \cdot \underline{a_{s n}^{H}}+\tilde{\tilde{\xi}_{s n}} \cdot \underline{a_{s n}^{\prime}} \cdot \underline{a_{s n}^{H}}\right)+\tilde{\sigma}^{2} \cdot \underline{\underline{1}}$

where each component of the vector (aisn $)_{q}$ is of the form:

$$
\left(\underline{a}_{s n}\right)_{q}=\left(\hat{a}_{s n}\right)_{q} \cdot\left(j \cdot k_{q} \cdot \cos \hat{\xi}_{s}\right) \cdot \hat{c_{n s}}
$$

The covariance error measure to be used hereafter will be just the first column of the resulting error matrix. This is equivalent to select a reference sensor and minimize the difference between the measured and the model crossspectrum between the reference sensor and the rest of sensors forming the aperture. The formulation of this measure will be:

$\left.\underline{\underline{\varepsilon}}=\tilde{\tilde{C_{x}}} \cdot 1=\Sigma_{s=1}^{N} \tilde{c_{s n}} \cdot \hat{a}_{s n}+\tilde{\zeta}_{s n} \cdot \underline{a}_{s n}\right)+\tilde{\sigma}^{2} \cdot 1$

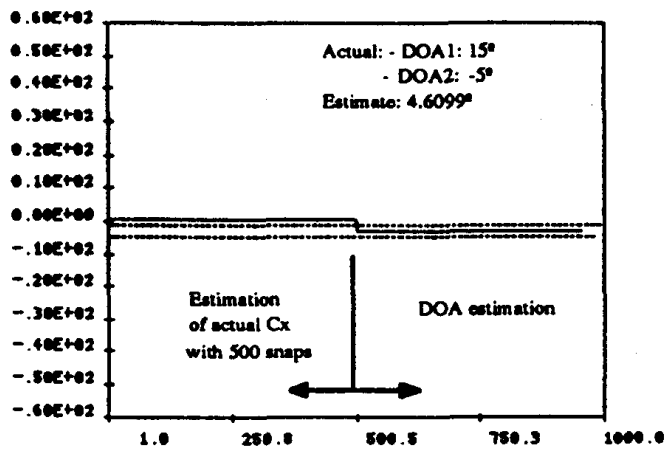

Figure 1

$1=(1,0,0, \ldots 0)^{\top}$

This result is of capital importance in deriving the corresponding linear filter, since it stablishes the fundamental relationship between the measured error and the parameter error

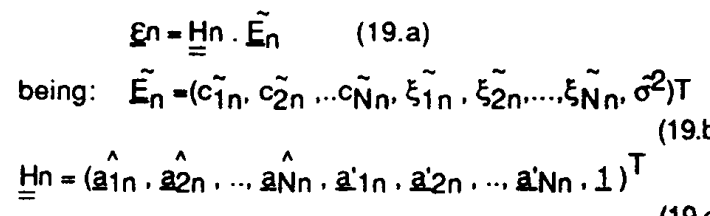

\section{LINEAR ADAPTIVE FILTER.}

Because of lack of space, here will be reported only a summary of the procedure. The presented simmulations form part of a complete set of results coverning the adaptive behaviour of the algorithm and the resulting performance.

The basic updates of the parameter estimates are done with the so-called gain matrix K:

$$
E_{n+1}^{n}=E_{n}+K_{n} \cdot \varepsilon_{n}
$$

Assuming that the actual paremeter vector $E$ remains stationary with the iterations plus an innovation noise with covariance matrix $\underline{\underline{Q}}$ assumed diagonal, the orthogonality between the parameters error $E_{n+1}^{\tilde{1}}$ and the measurement error En provides the optimum gain matrix:

$\underline{\underline{K} n}=\underline{\underline{\Sigma}} n \cdot \underline{\underline{H}} n^{H} \cdot \underline{\underline{H}} n \cdot \underline{\underline{\Sigma}} n \cdot \underline{H}_{n} H^{-1}=\underline{\underline{\Sigma}} n \cdot \underline{H}^{H} \cdot \underline{\zeta}^{-1}$

Matrix $\sum n$ is defined as the parameters covariance at iteration $n$. The update of this matrix is based also in the stationary evolution of the actual parameters vector and the covariance of the innovation noise. The resulting update is: 


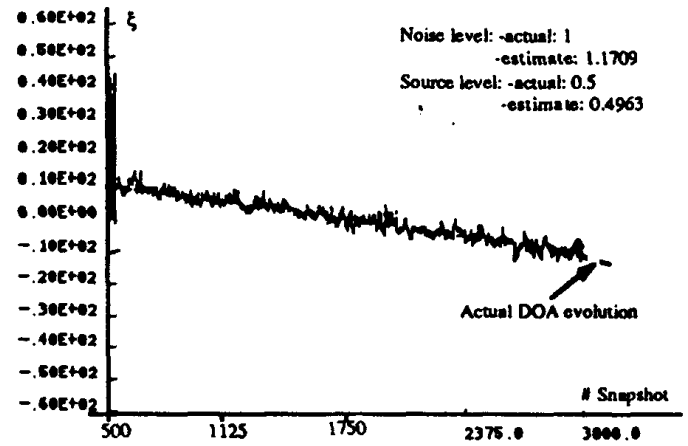

Figure 2.

$$
\underline{\underline{\Sigma}} n+1=\underline{\underline{\Sigma}}-\underline{\underline{K}} \mathrm{E} \cdot \underline{\zeta_{n}} \cdot \underline{\underline{K} \mathrm{n}^{H}}+\underline{\underline{Q}}
$$

As an example of the ML behaviour of this fastconvergence algorithm, figure 1 shows the case when the actual number of sources that are present in the scenario (e.g.:2 sources) does not match the number of sources set in the model (e.g:1 source). The resulting DOA estimate is quite close the arithmetic mean of the actual ones. The other presented simulation in figure 2 shows how the mall error approximation can cope with the problem of simultaneous tracking of magnitude and DOA; the algorithm provides a good tracking of the moving source (from $10^{2}$ to $-10^{\circ}$ ) in spite of the low SNR $(-3 \mathrm{~dB}$ 's) and the small aperture considered. In both figures, the aperture considered was formed by 7 sensors and the covariance matrix $\underline{\underline{\underline{C}}} \times$ was calculated from only 500 snapshots.

\section{CONCLUDING REMARKS.}

Using the small error approximation, it has been reported that a measurement of the covariance error matrix can be formulated in a linear dependence of the parameter error vector. This has been useful in order to design a Kalman like algorithm. This one provides a fast estimation of the source parameter, that behaves in a ML manner, even if the actual date covariance matrix is computed from short records of aperture snapshots. The range of possibilities this method offers is wide. More simulations have been made and, as expected, they have proved that this fitter enjoys of the main Kalman filter's features. That is on the one hand, its computational ease and the tracking capability makes it a good choice for narrowband spatial reference beamforming applications, on the other, it offers an accurate and robust estimates for closely located sources and small apertures, whatsoever initial parameter guess. All these are features, the existing signal estimation methods do not offer.

Finally, we point out that as a topic for further research we are investigating the way of simultaneously tracking the DOA and magnitude of two signals in noise by means of a system composed by two linear adaptive filters in parallel, with feedback from the output of each filter to the input of the other one.

\section{REFERENCES.}

[1] B.D.O. Anderson and J.B. Moore, "Optimal Filtering," Englewood Cliffs, N.J. Prentice-Hall, 1979.

[2]W.S.Liggett, "Passive Sonar:Fitting Models to Muttiple Tme Series," in Signal Processing, Griffiths, J.W.R. et al. ods., Academic Press, New York, 1973, pp.327-345.

[3]J.F.Böhme. "Separated Estimation of Wave Parameters and Spectral Parameters by Maximum Likelihood," IEEE proceedings 1986, 52.7.1-52.7.4, pp.2819-2822.

[4] M.Wax, T.Kailath, "Optimum Localization of Multiple Sources by Passive Arrays," IEEE tras. 1983, Vol.ASSP-31, no.5, pp. 1210-1218.

[5] Y.Bresler, A.Macovski, "Exact Maximum Likelihood Parameter Estimation of Superimposed Exponential Signals in Noise," IEEE Trans.1986, Vol. ASSP-34, no.5, pp.1087-1089.

[6] H.Watanabe, M.Suzuki, Maximum Likelihood Bearing Estimation by Quasi-Newton Method using a Uniform Linear Array," IEEE proceeding 1991, p.p. 3325-3328.

17)J.F.Böhme, "Estimation of Spectral Parameters of Correlated Signal in Wavefields," Signal Processing 1986, Elsevier Science Publixhers,p.p. 329-337.

[8] D.Kraus, G.Schmitz, J.F.Böhme, "Least Squares Estimates for Source Location and Asymptotic Behaviours, " in Signal Processing V . Elsevier Science Publishers, 1990, p.p. 649-652

[9] H.A. d'Assumpcao, "Some new Signal Processors for Arrays of Sensors," IEEE Transactions on Information Theory, Vol. IT-26, no.4, July 1980, p.p.441-454.

[10] M.Basseville, "Distance Measures for Singal Processing and Pattern Recognition, "Signal Processing 1989, Elsevier Science Publishers, p.p. 349-369. 